\title{
A brief review of experiences with short-term clinical trials monitored by mouse-foot-pad inoculation*
}

\author{
C C SHEPARD \\ Leprosy and Rickettsia Branch, Virology Division, Centre for \\ Infectious Diseases, Centres for Disease Control, Department \\ of Health and Human Services, Atlanta, Georgia, 30333, USA
}

Received for publication 8 April 1981

\begin{abstract}
Summary Beginning in 1964 we used mouse-foot-pad inoculations to monitor the loss in numbers of viable Mycobacterium leprae that occurs when the patient begins antileprosy therapy. Our studies eventually involved patients at the US Public Health Service Hospital in San Francisco and the Leonard Wood Memorial facilities in Cebu, Philippines and mouse-foot-pad laboratories in San Francisco and Cebu, as well as Atlanta. We found that to monitor short-term therapeutic trials, the mouse-foot-pad method was the most efficient one available, in the sense that it required the smallest number of patients. All the results are compiled here in a standard form of presentation to facilitate comparisons between trials and regimens. A table is provided for statistical consideration of results such as these.
\end{abstract}

\section{Introduction}

Most of the results to be presented have already been published, but their collection in one place in a standard form may be helpful. Beginning in 1964 Levy and I, in collaboration with Fasal, (Levy and Fasal were then at the US Public Health Service Hospital in San Francisco) began to employ mouse-footpad inoculations to monitor the decrease in numbers of viable Mycobacterium leprae that occur when the patient begins antileprosy therapy. We already knew from the earliest mouse-foot-pad days that the bacilli from untreated patients were regularly infective for mice and that those from patients who had been

* Originally presented at the October 1980 meeting of the THELEP Scientific Working Group in Geneva, at which it was agreed to submit papers for publication in both Leprosy Review and International Journal of Leprosy. It is our understanding that similar publications will be printed in the December 1981 issue of $I J L$. Editor 
treated with sulphones for months or years were not, and we were curious about the timing of the loss of detectable viability. All of the work could have been carried out in San Francisco, but by doing the inoculations in Atlanta we could ensure a collaboration that we correctly sensed would be productive.

Eventually we found that with mouse-foot-pad inoculations the early loss in viability of $M$. leprae could be followed much more satisfactorily than with existing methods. True, 12 months had to pass before a negative result could be registered, but we were measuring the solid ratios (morphological indices, MIs) of the M. leprae in the specimens at the same time anyhow. With measurements of the solid ratios we could monitor a decrease in number of viable $M$. leprae by about $90 \%$ (conveniently expressed as a loss of one 'log' because the logarithm to the base of 10 of the number of viable $M$. leprae is reduced by one). With mouse inoculation we would monitor two or three logs of loss and could do so with more accuracy near the end point. Thus, it seemed clear that this was the most efficient available method of monitoring short-term trials in man. Later, under the United States-Japan Cooperative Medical Science Program, it became possible to start clinical trials in collaboration with Leonard Wood Memorial personnel in Cebu, Philippines, and a mouse-foot-pad facility was developed there. These trials started in 1969 and continued until administrative difficulties led to their loss in 1974.

\section{Materials and Methods}

Skin-punch biopsy specimens $(5-6 \mathrm{~mm})$ were removed at appropriate intervals, a tissue suspension prepared, and the bacilli counted. Usually, 20 mice were inoculated. The inoculum was diluted, if necessary, to contain not more than $5 \times 10^{3} \mathrm{M}$. leprae. CFW mice were used in Atlanta, BALB/c in San Francisco, and usually CBA in Cebu. Systematic comparisons failed to show differences between CFW and BALB/c, or between BALB/c and CBA. Biopsy specimens from San Francisco patients were shipped by air to Atlanta, and those from Cebu patients were divided in two for parallel inoculation in San Francisco and Cebu in the beginning. Later, only a sample of the Cebu specimens were shipped to San Francisco. From 3 through 12 months after inoculation, a mouse was taken each month for histological sections. When a section contained acid-fast bacteria (AFB), four mice were taken for counts of AFB in their pooled foot-pad tissues. In San Francisco and Cebu, additional harvests were frequently carried out at about 12 months if all the sections were negative.

With these data, one can calculate the incubation period and generation time and construct an $M$. leprae killing curve for the drug in each patient. Of course, the lepromatous patient has a total of $10^{11}$ or $10^{12}$ viable M. leprae in his tissues and we are following only the initial two or three logs of the killing in this type of short-term trial. The most important point of this M. leprae 
killing curve is the lowest point we can detect; that is, the time when the number of viable $M$. leprae decreases to subdetectable numbers. Consequently, the most important feature of the response in each patient can be shown by two points, the time of the last positive specimen and the time of the next negative specimen. In the figures each line represents a patient and the two symbols on each line represent these two critical times. Results are not shown when the inoculum contained less than $1 \times 10^{3} \mathrm{AFB}$, or when the pretreatment specimen gave weak or negative results in the mice.

For the statistical analysis in the later trials where the specimens were taken at regular times after treatment, the comparisons could be made between the number of specimens positive or negative at particular times. In the early trials, however, when the optimum times for collection were not yet known, the patients were counted as positive any time before the last positive specimen and as negative any time after that (even though the next negative specimen had not yet been taken). When a negative specimen was never obtained (marked with question marks at the end of a patient's line), the patient was included in the analysis only for the times before the last positive specimen. The times selected for analysis were determined by the speed of response to a regimen. For example, in Figure 1 the results were analysed at 50, 100 and 150 days. For all trials the differences between regimens were analysed by Fisher's exact test, specifically, for the time of treatment to be analysed a fourfold table was constructed to show the number of positive and negative patients for the two regimens. The $P$ values given are for a one-tailed test. Of course, when the patients are not assigned to regimen by random selection there is an increased risk that factors other than differences in drug regimen are responsible for the observed differences between groups. Nevertheless, the analysis determines what the probabilities are that the difference observed would occur by chance alone.

\section{Results}

The early results in San Francisco 1, 2, 3 are presented in Figure 1. The B663 and the ethionamide patients had received earlier sulphone therapy and were in relapse. The others were new patients. There was no attempt to randomize the assignment of patients to regimen. The differences between regimens were analysed statistically at 50,100 and 150 days. All of the differences between groups could be explained as statistical variation except those between DDS and DADDS at 100 and 150 days.

We then turned to rifampicin in a series of trials, starting in 1970. (Figure 2) The first was a randomized trial comparing $600 \mathrm{mg}$ rifampicin with $50 \mathrm{mg}$ DDS daily. ${ }^{4,5}$ The DDS patients are included in Figure 1. Note that the time scale in Figure 2 is changed. Rifampicin killed M. leprae so rapidly that the difference 


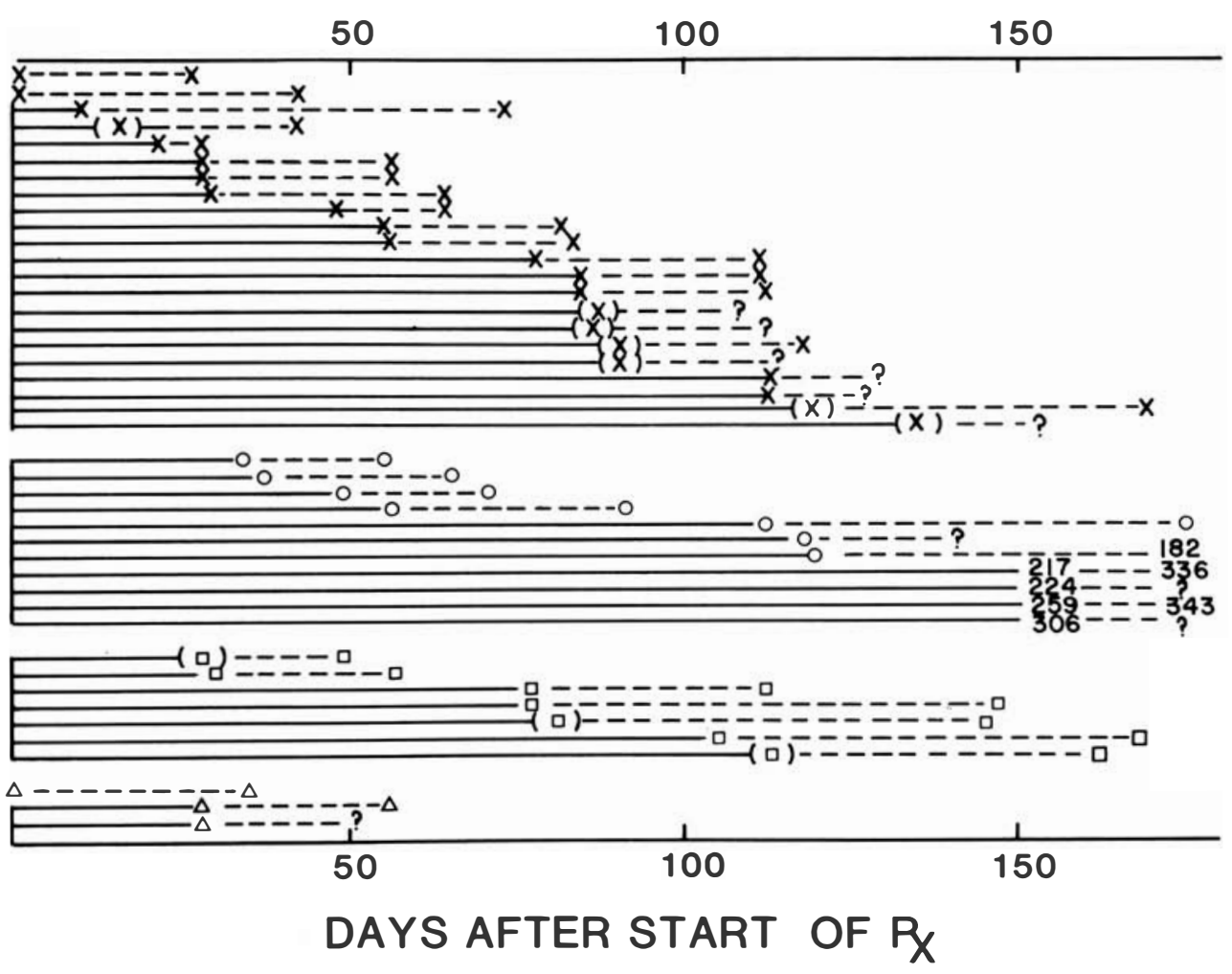

Figure 1. Results of mouse-foot-pad inoculation of $M$. leprae from serial skin-punch biopsy specimens taken from lepromatous patients in San Francisco. ${ }^{1,2,3}$ Each line represents a patient and the two symbols represent only the last positive specimen (last specimen with $M$. leprae infective for mice) and the next negative specimen. Parentheses indicate a weakly positive result; that is, either the monthly histological section or the harvest (count of AFB in a pool of usually four mice) was negative. A single parenthesis indicates a harvest less than $1 \times 10^{5}$. A question mark indicates that the last specimen taken was still positive. DDS $=$ dapsone, DADDS $=$ acedapsone, $\mathrm{B} 663=$ clofazimine, $\mathrm{ETH}=$ ethionamide. $\mathrm{x}, \mathrm{DDS}$ to $50 \mathrm{mg} / \mathrm{d}$; O, DADDS, $225 \mathrm{mg} / 77 \mathrm{~d}$; $\square$, B $663,200 \mathrm{mg} / \mathrm{d}$; $\triangle$, ETH, $259 \mathrm{mg}$ tid.

was dramatic, with no overlap between the rifampicin and DDS results at 14 days. The $P$ value here is $\ll 0.001$.

The second rifampicin trial in San Francisco was a trial of a single dose of $1,500 \mathrm{mg}^{5,6}$ (Figure 2). Actually the regimen included $10 \mathrm{mg}$ DDS daily, but it seems very unlikely that the DDS had detectable effect by 3-5 days, the time the second specimen was taken.

Next there was a comparison of single doses of 600,900 and $1,200 \mathrm{mg}$ rifampicin with randomized assignment of patients to treatment group. ${ }^{7}$ The differences between the groups were not significant, but in view of the previous experience with larger intakes, it is probably important that one of the patients 


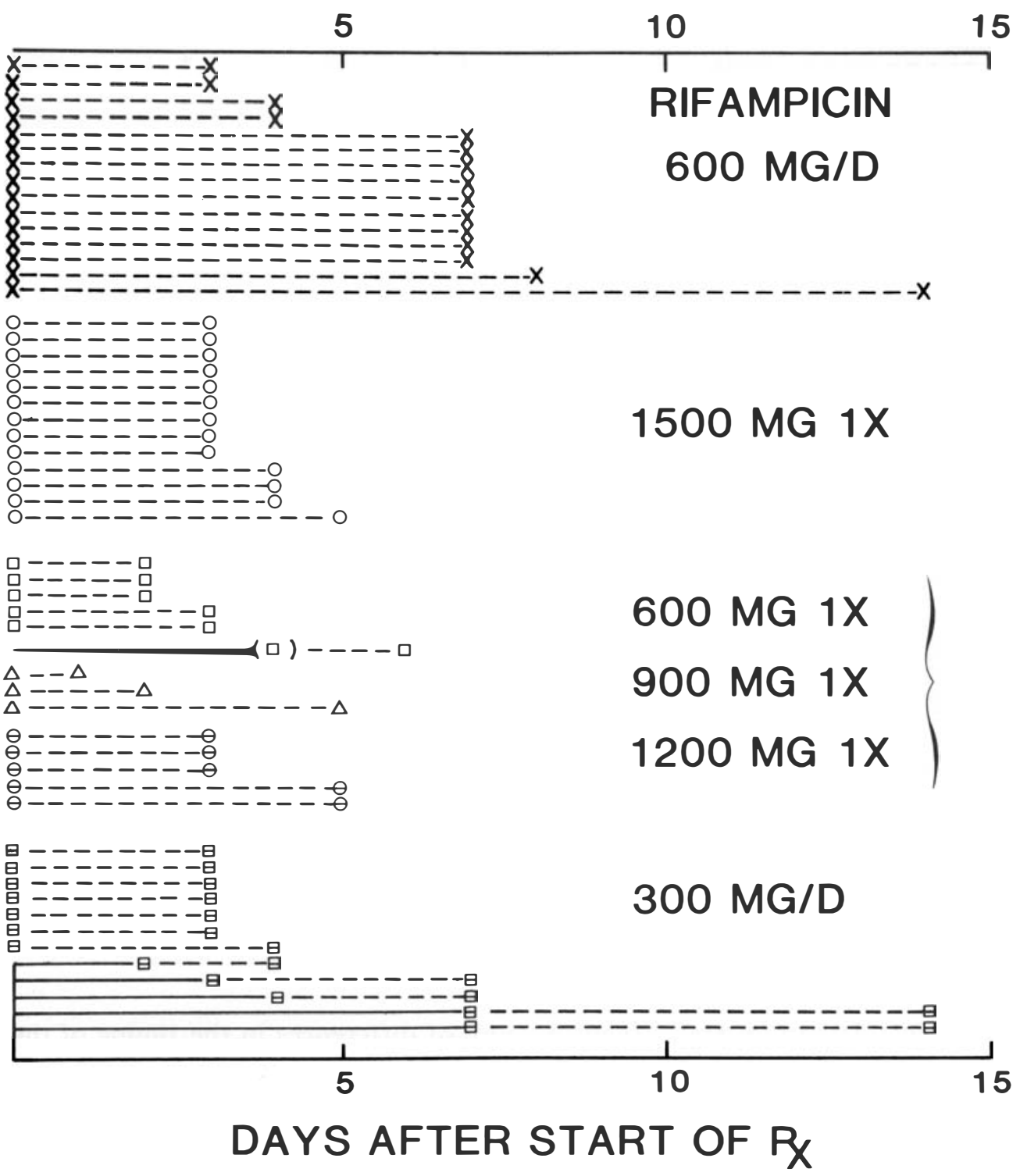

Figure 2. Results with rifampicin treatment in San Francisco. ${ }^{4-7}$

receiving $600 \mathrm{mg}$ had a weakly positive result at 4 days (positive section but negative harvest, which indicated that the inoculum contained very few viable bacilli).

Finally, in an attempt to reach the end point for rifampicin, we tried $300 \mathrm{mg}$ a day. ${ }^{7}$ Here 5 of 17 patients had positive specimens after the start of treatment, so that these patients had received a total of $300-1,800 \mathrm{mg}$ rifampicin before the last positive specimen was collected. Comparisons with 


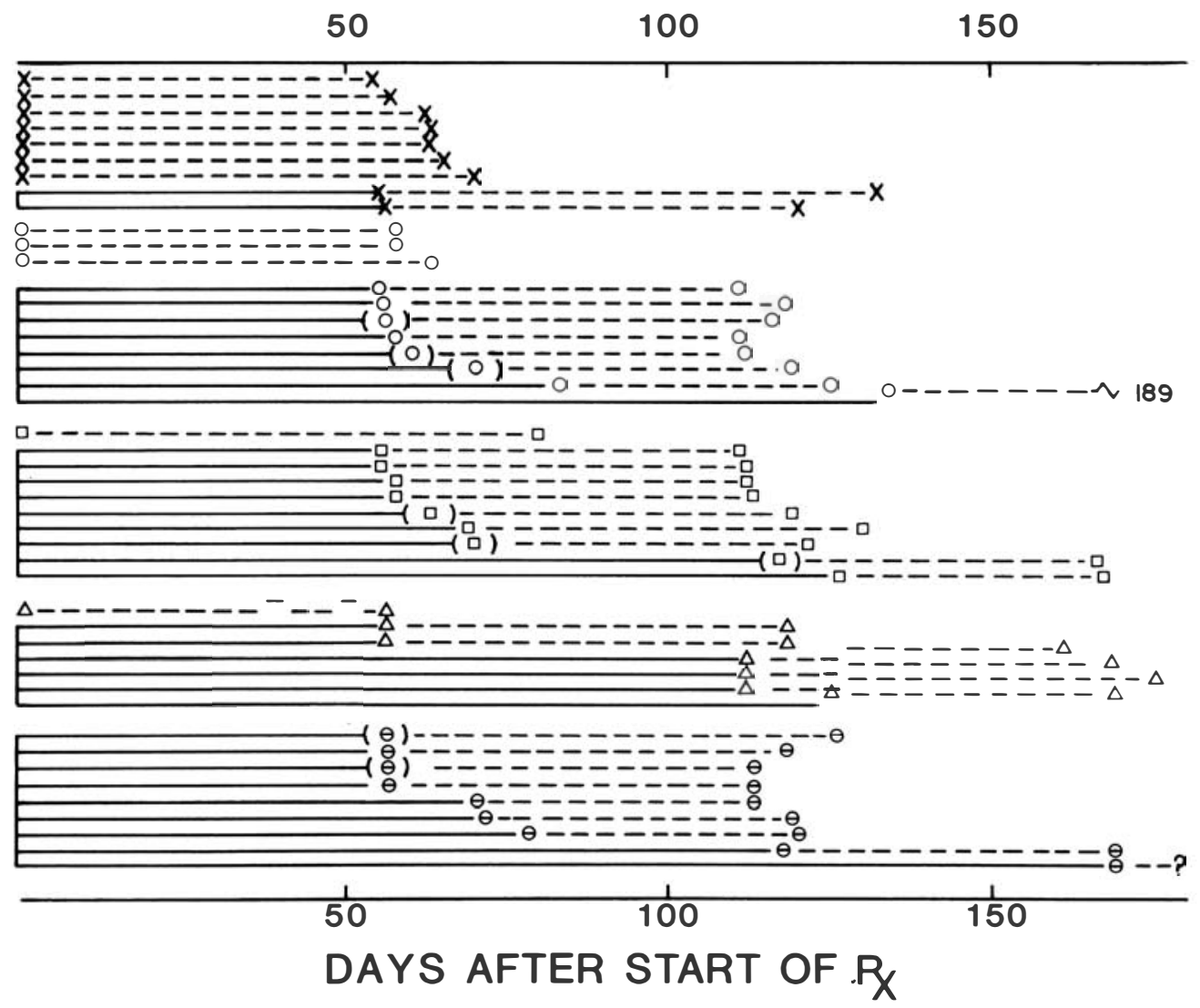

Figure 3. Results with $\mathrm{B} 663$ trial in Cebu. ${ }^{8}$ B663 II trial: $\mathrm{x}, 200 \mathrm{mg}, 6$ times per week; $\circ, 100 \mathrm{mg} 6$ times per week; $\square, 300 \mathrm{mg}$ once a week; $\triangle, 600 \mathrm{mg}$, once every 2 weeks; $\ominus, 600 \mathrm{mg}$, 2 consecutive days Q 4 weeks.

the $600 \mathrm{mg} /$ day group are difficult because of differences in the timing of the collection of specimens. A comparison with the single dose $600 \mathrm{mg}$ group is possible, but the differences are not statistically significant.

The results of the first trial in Cebu monitored in this way ${ }^{8}$ are shown in Figure 3. Regimen I was $200 \mathrm{mg}$ B663 6 days a week and the others involved t-week totals of 1,200 mg, spaced at various intervals. By inspection, Regimen I appears to be more effective than the others, and the differences among Regimens II, III, IV and V appear to be small. Because the patients were randomly assigned to the five regimens, we may be more confident about the meaning of statistically significant differences. At 50 days, the results with Regimen I are significantly different from those with other groups (for I vs II, $P=0.035$ ), but the differences among II, III, IV and V are far from significant. At 100 days, the only significant differences are between I and IV $(P<0.019)$ and II and IV $(P<0.047)$. The differences between the combined II and III and combined IV and $\mathrm{V}$ are not significant at either interval. 


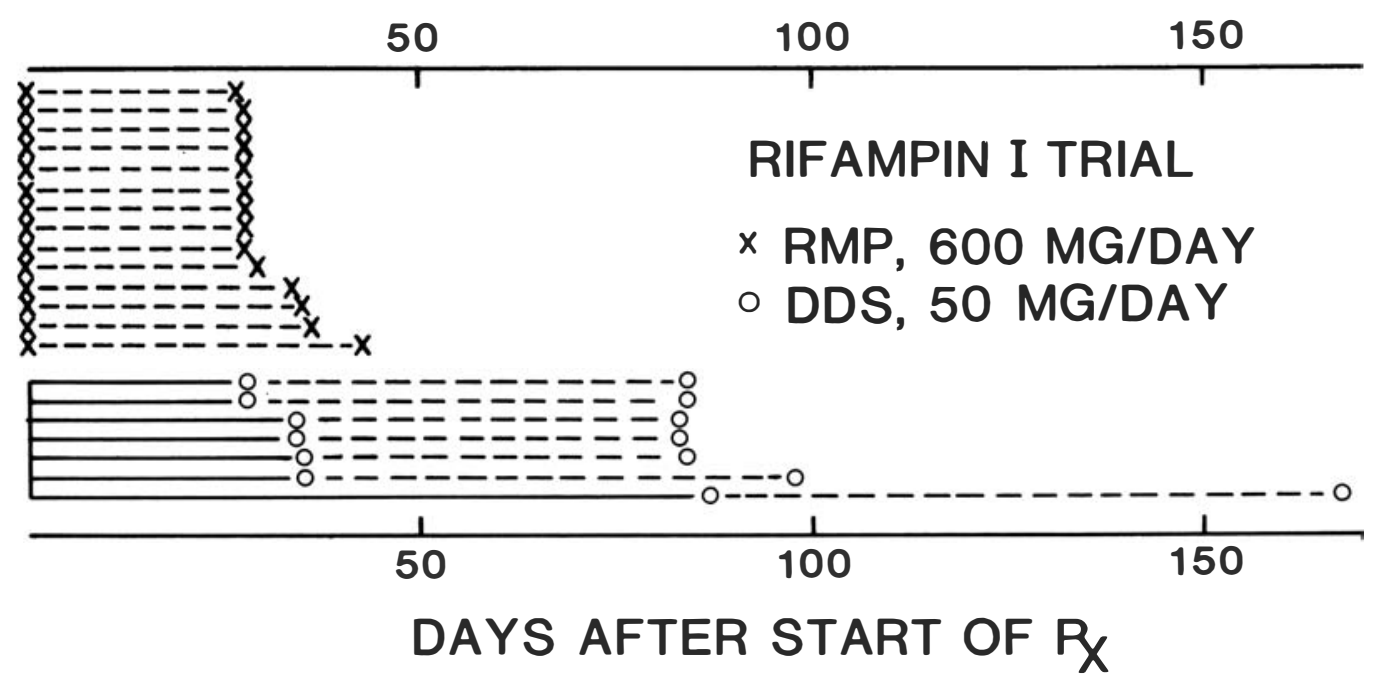

Figure 4. Results with Rifampin I trial in Cebu. ${ }^{9}$

The next use in Cebu was the Rifampin trial ${ }^{9}$ (Figure 4), which compared the daily administration of rifampicin to DDS, with patients assigned to regimen on a randomized basis. As one would expect, the difference was marked $(P \ll 0.001)$.

The final use in Cebu under the US-Japan Cooperative Medical Science Program was the Rifampin II trial (unpublished results) (Figure 5), in which there were three groups: DADDS alone, DADDS $+1,500 \mathrm{mg}$ rifampicin at the time of the DADDS injection, and $600 \mathrm{mg}$ daily rifampicin. There were no detectable differences between the second and third groups, but as expected, the differences between the first group and the other two were marked (for 1 vs $2, P \ll 0.001$; for 1 vs $3, P \ll 0.001$ ).

The reader might wonder why the Rifampin I and II trials were so large when they were comparing regimens whose efficacy had already been studied in short-term trials. In fact, the Rifampin I and II trials were designed to supply patients for trials of maintenance regimens by long-term followup. The longterm maintenance regimens involved comparisons between DADDS and DDS, with visits to the clinic every 12 weeks. Rifampin I started in early 1971 and Rifampin II in late 1971, so if the trials had not been lost (because of administrative difficulties) they would now be supplying information of critical value to leprosy care.

\section{Discussion}

This has been a brief review of short-term leprosy trials monitored by mousefoot-pad inoculations. A simplified method for presenting the data was used to facilitate comparisons of all the trials to date.

In examining the results obtained with a single regimen, one is struck by 


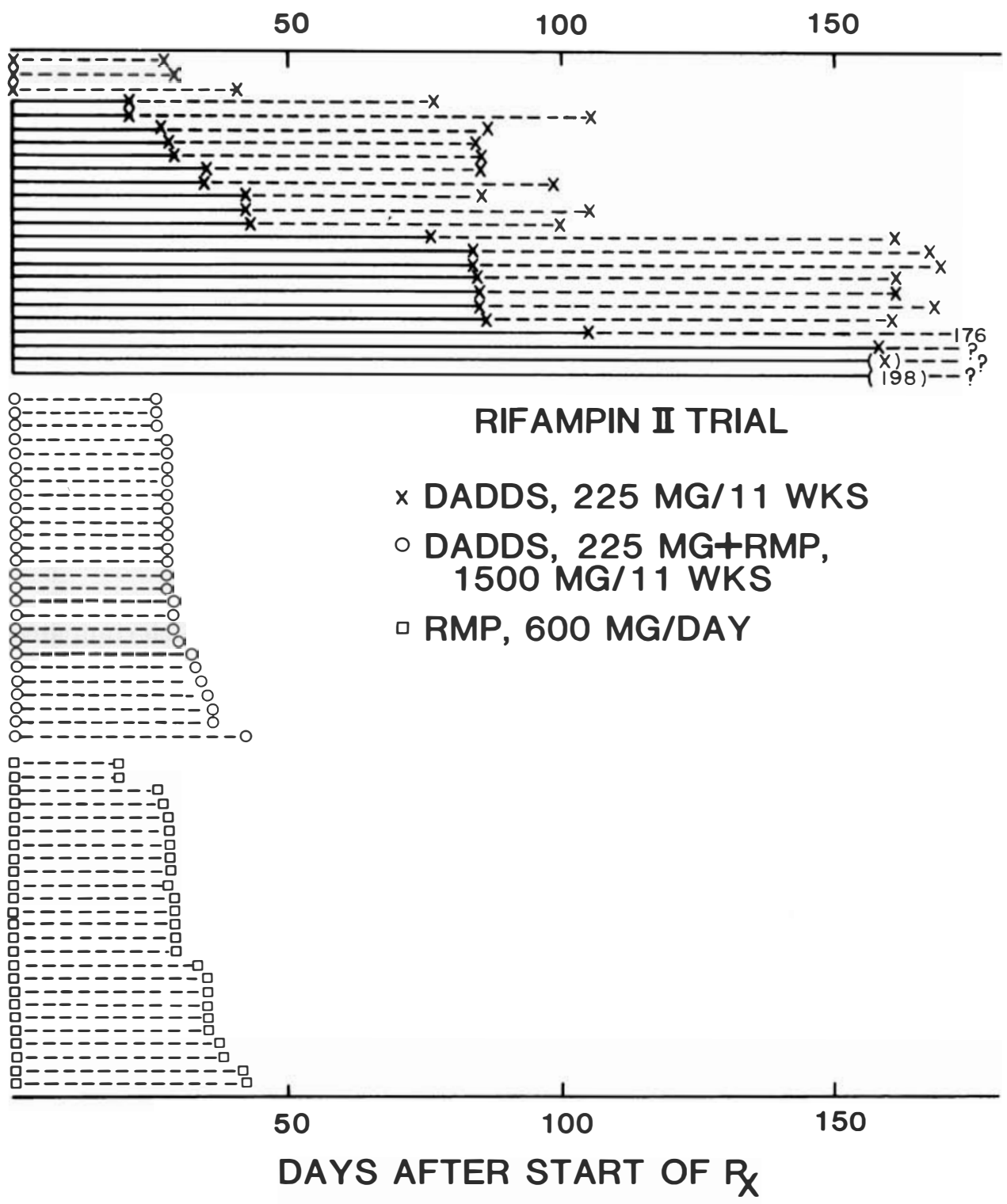

Figure 5. Results with Rifampin II trial in Cebu (Collaborative effort, unpublished).

the large differences in rate of response between different patients. These differences do not appear to be caused by differences in the determined sensitivity of the patient's $M$. leprae to drug. Thus, in the DADDS trial in San Francisco the $M$. leprae from the last positive specimen from the slowest responding patients were shown to be fully sensitive to DDS $(0.0001 \%$ in the mouse diet), and in the Rifampin II trial some of the isolates from the patients responding most slowly to DADDS were found sensitive to $0.00003 \%$ and 
Table 1. Critical ${ }^{\mathrm{a}} P$ values by Fisher's exact test in fourfold tables ${ }^{\mathrm{b}}$.

\begin{tabular}{|c|c|c|c|c|c|c|c|c|c|}
\hline$a+b$ & $c+d$ & $\mathrm{a}$ & $\mathrm{c}$ & $P$ & $a+b$ & $c+d$ & $\mathrm{a}$ & $\mathrm{c}$ & $P$ \\
\hline 3 & 3 & 0 & 3 & 0.050 & 10 & 10 & 0 & 10 & 0.000005 \\
\hline \multirow[t]{2}{*}{4} & 4 & 0 & 4 & 0.014 & & & 1 & 10 & 0.00006 \\
\hline & & & & & & & 2 & 10 & 0.0004 \\
\hline \multirow[t]{2}{*}{5} & 5 & 0 & 5 & 0.004 & & & 3 & 10 & 0.001 \\
\hline & & 0 & 4 & 0.024 & & & 4 & 10 & 0.005 \\
\hline \multirow[t]{8}{*}{6} & 6 & 0 & 6 & 0.001 & & & 5 & 10 & 0.016 \\
\hline & & & 5 & 0.008 & & & 6 & 10 & 0.043 \\
\hline & & & & & & & 0 & 9 & 0.00006 \\
\hline & & & & & & & 1 & 9 & 0.005 \\
\hline & & & & & & & 2 & 9 & 0.003 \\
\hline & & 1 & 5 & 0.040 & & & 3 & 9 & 0.010 \\
\hline & & 0 & 4 & 0.030 & & & 4 & 9 & 0.029 \\
\hline & & & & & & & 0 & 8 & 0.0004 \\
\hline \multirow[t]{6}{*}{7} & 7 & 0 & 7 & 0.0003 & & & 1 & 8 & 0.003 \\
\hline & & 1 & 7 & 0.002 & & & 2 & 8 & 0.012 \\
\hline & & 0 & 6 & 0.002 & & & 3 & 8 & 0.035 \\
\hline & & & & & & & 0 & 7 & 0.002 \\
\hline & & 1 & 6 & 0.015 & & & 1 & 7 & 0.010 \\
\hline & & 0 & 5 & 0.010 & & & 2 & 7 & 0.035 \\
\hline \multirow[t]{8}{*}{8} & 8 & 0 & 8 & 0.00008 & & & 0 & 6 & 0.005 \\
\hline & & 0 & 7 & 0.0007 & & & 1 & 6 & 0.029 \\
\hline & & 1 & 7 & 0.005 & & & 0 & 5 & 0.016 \\
\hline & & 2 & 7 & 0.020 & & & 0 & 4 & 0.043 \\
\hline & & 0 & 6 & 0.003 & & & & & \\
\hline & & 1 & 6 & 0.020 & & & & & \\
\hline & & 0 & 5 & 0.013 & & & & & \\
\hline & & 0 & 4 & 0.038 & & & & & \\
\hline
\end{tabular}

${ }^{\text {a }}$ Includes all instances where $P \leqslant 0.05$ by one-tailed test.

${ }^{\mathbf{b}}$ Tables constructed as follows:

$\begin{array}{lccc} & \text { Positive }^{c} & \text { Negative }^{c} & \\ \text { Regimen 1 } & \mathrm{a} & \mathrm{b} & \mathrm{a}+\mathrm{b} \\ \text { Regimen 2 } & \mathrm{c} & \mathrm{d} & \mathrm{c}+\mathrm{d}\end{array}$

${ }^{\mathbf{c}}$ Interchange positive and negative columns if necessary to provide fit to the table.

$0.00001 \%$ DDS, dosages producing about 3 and $1 \mathrm{ng}$ of DDS, respectively, in the plasma of the mice. Because of these unaccountable variations between patients, statistical analysis needs to be applied to the data. The method we have used here is simple. The calculations involve factorials, but tables are widely available, for example, in older editions of the Handbook of Chemistry and Physics and some, even inexpensive, hand calculators have a key for factorials. Moreover, the formula can be programmed into certain, moderately priced, hand calculators.

Table 1 is provided for results with (equal) group sizes of $3-10$. The use of the table is given in its footnote. As an example, we might consider a comparison of two groups of 10 patients in which, at a particular interval, 'regimen 
1' gave 0 positive and 10 negative results and 'regimen 2' gave 6 positive and 4 negative results. In the fourfold table shown, there would be the following entries: for $\mathrm{a}, 0 ; \mathrm{b}, 10 ; \mathrm{a}+\mathrm{b}, 10 ; \mathrm{c}, 6 ; \mathrm{d}, 4 ; \mathrm{c}+\mathrm{d}, 10$. The one-tailed test may be used when the difference is in the expected direction; in this example, $P$ would be $<0.043$. The two-tailed test must be used when one cannot predict which drug will be more effective; here $P$ would be twice the value shown in the table, or $<0.086$.

Of the available procedures for short-term trials, the foot-pad method is the most efficient, in the sense that it needs the fewest patients. The number of patients required for a test of a new drug cannot be set without knowing the activity of the drug, and when the drug is new, the information may be limited to results from studies in mice, for example, by the kinetic method or the proportional bactericidal method. The group size needed can be estimated with the aid of Table 1. With two drugs that have as different short-term activity as DDS and rifampicin, only two groups of three patients are needed to discriminate with a $P$ value of $<0.05$. If there is apt to be some overlap between the results with the two drugs, and it is important to detect a statistically significant difference between the drugs, and group sizes need to be at least 6-10.

The timing of the specimens varies with the drugs. For rifampicin, we learned from kinetic method results in mice that the second specimen could be taken as early as $2-3$ days. For drugs with a short-term killing rate similar to that of DDS, a schedule of $0,4,8$ and 12 weeks would probably be suitable.

\section{References}

1 Shepard CC, Levy L, Fasal P. The death of Mycobacterium leprae during treatment with 4,4'-diaminodiphenylsulfone (DDS). Am J Trop Med Hyg, 1968; 17: 769-75.

2 Levy L, Shepard CC, Fasal P. Clofazimine therapy of lepromatous leprosy caused by dapsone-resistant Mycobacterium leprae. Am J Trop Med Hyg, 1972; 21: 315-21.

3 Shepard CC, Levy L, Fasal P. The death rate of Mycobacterium leprae during treatment of lepromatous leprosy with acedapsone (DADDS). Am J Trop Med Hyg, 1972; 21 : 440-5.

4 Shepard CC, Levy L, Fasal P. Rapid bactericidal effect of rifampin on Mycobacterium leprae. Am J Trop Med Hyg, 1972; 21: 446-9.

5 Shepard CC, Levy L, Fasal P. Further experience with the rapid bactericidal effect of rifampin on Mycobacterium leprae. Am J Trop Med Hyg, 1974; 23: 1120-4.

6 Shepard CC, Levy L, Fasal P. Rifampin's rapid bactericidal action on M. leprae. Ain Shams Med J, 1974; 25: 279-86.

7 Levy L, Shepard CC, Fasal P. The bactericidal effect of rifampicin on M. leprae in man: (a) single doses of 600,900, and $1200 \mathrm{mg}$; and (b) daily doses of $300 \mathrm{mg}$. Int J Lepr, 1976; 44: 183-7.

8 Collaborative effort of the U.S. Leprosy Panel (U.S.-Japan Cooperative Medical Science Program) and the Leonard Wood Memorial: Spaced clofazimine therapy of lepromatous leprosy. Am J Trop Med Hyg, 1976; 25: 434-7.

9 Collaborative effort of the U.S. Leprosy Panel (U.S.-Japan Cooperative Medical Science Program) and the Leonard Wood Memorial: Rifampin therapy of lepromatous leprosy. Am J Trop Med Hyg, 1975; 24: 475-84. 